\title{
$k$-Contra Harmonic Mean Labeling of Snake Related Graphs
}

R Gopi

${ }^{1}$ Department of Mathematics, Srimad Andavan Arts and Science College (Autonomous), Tiruchirappalli - 620 005, Tamilnadu, India

Email: drrgmaths@gmail.com

ARTICLE INFORMATION

Received: 17 September, 2018

Revised: 11 October, 2018

Accepted: 06 February, 2019

Published online: March 6, 2019

Keywords:

$k$-Contra harmonic labeling, $k$-Contra

harmonic graph, Triangular snake, Double

Triangular snake.

DOI: https://doi.org/1015415/mjis.2019.72010

\section{ABSTRACT}

A graph $G(p, q)$ is said to have a $k$-Contra Harmonic mean labeling if there exists an injection $f: V \rightarrow\{k-1, k, k+1, \ldots, k+q-1\}$ such that the induced map $f$ is defined by $f^{*}(u v)=\left\lceil\frac{f(u)^{2}+f(v)^{2}}{f(u)+f(v)}\right\rceil$ or $\left\lfloor\frac{f(u)^{2}+f(v)^{2}}{f(u)+f(v)}\right\rfloor$ is a bijection from E to $\{k, k+1, \ldots, k+q-1\}$. A graph that admits a $k$-Contra Harmonic Mean Labeling $(\mathrm{k}$ CHML) is called $k$-Contra Harmonic Mean Graph(k-CHMG).

\section{Introduction}

In (Narasimhan et. al. 2013) introduced the Contra Harmonic mean labeling. In this paper we prove that some snake related graphs. Throughout this paper $k$ denote any positive integer $>0$. For all other terminology and notations we follow (Harary 1988), (Gallian, 2018).

\section{Main Results}

\section{Theorem 2.1}

The graph $T S_{m}(m \geq 3)$ is a $K$-Contra Harmonic mean graph for any $k$.

\section{Proof}

Let $\left\{u_{l}, 1 \leq l \leq m, v_{l}, 1 \leq l \leq m-1\right\}$ be the vertices and $\left\{a_{l}, b_{l}, c_{l}, 1 \leq l \leq m-1\right\}$ be the edges which are denoted as in Figure 2.1.

First we label the vertices:

For $1 \leq l \leq m-1 f\left(v_{l}\right)=k+3 l-3$

For $1 \leq l \leq m \quad f\left(u_{l}\right)=k+3 l-4$

Then the induced edge labels are:

$$
f^{*}\left(a_{1}\right)=k+2
$$

For $2 \leq l \leq m-1 \quad f^{*}\left(a_{l}\right)=k+3 l-2 f^{*}\left(c_{1}\right)=k+1$

$$
f^{*}\left(c_{l}\right)=k+3 l-1
$$

For $1 \leq l \leq m-1 \quad f^{*}\left(b_{l}\right)=k+3 l-3$

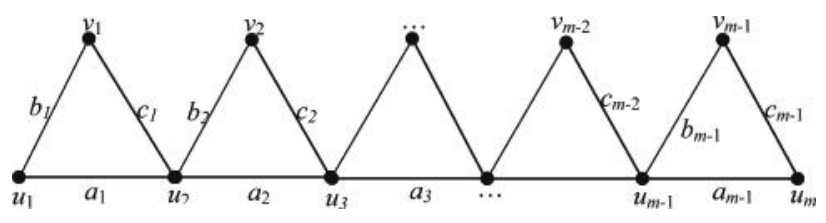

Figure 2.1. Ordinary labeling of $T S_{m}$

Hence, the edge labels are all distinct.

4-CHML of $T_{5}$ is shown in Figure 2.2.

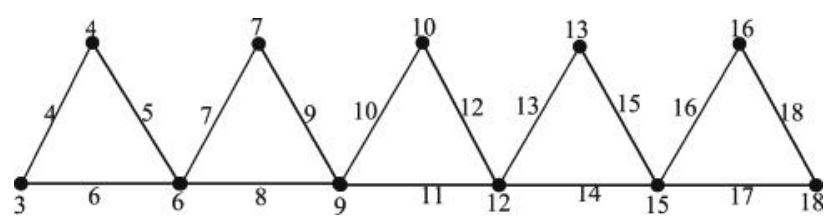

Figure 2.2: 4-CHML of $T S_{5}$

\section{Theorem 2.2}

The graph $A\left(T S_{m}\right)(m \geq 4)$ is a $k$-Contra Harmonic mean graph for any $k$. 


\section{Proof}

Let $\left\{u_{l}, 1 \leq l \leq m, v_{l}, 1 \leq l \leq \frac{m}{2}\right\}$ be the vertices and $\left\{a_{l}, 1 \leq l \leq m-1, b_{l}, c_{l}, 1 \leq l \leq \frac{m}{2}\right\}$ be the edges which are denoted as in Figure 2.3.

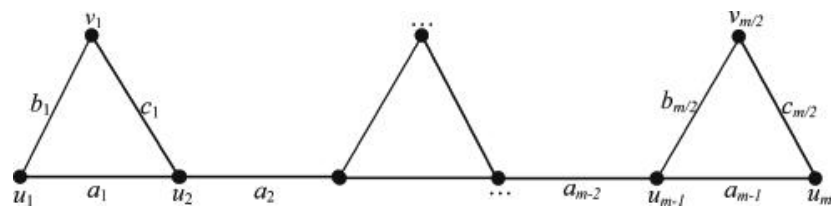

Figure 2.3. Ordinary labeling of $A\left(T S_{m}\right)$

First we label the vertices:

For $1 \leq l \leq \frac{m}{2} \quad f\left(v_{l}\right)=k+4 l-5$

For $1 \leq l \leq m \quad f\left(u_{l}\right)=k+2 l-2$

Then the induced edge labels are:

$$
f^{*}\left(a_{1}\right)=k+1
$$

For $2 \leq l \leq m-1$

$$
f^{*}\left(a_{l}\right)= \begin{cases}k+4 l-6 & \text { if } l \text { is odd } \\ k+2 l-1 & \text { if } l \text { is even }\end{cases}
$$

For $1 \leq l \leq \frac{m}{2} f^{*}\left(b_{l}\right)=k+4 l-4$

$$
f^{*}\left(c_{1}\right)=k+2
$$

For $2 \leq l \leq \frac{m}{2} f^{*}\left(c_{l}\right)=k+4 l-3$

Hence, the edge labels are all distinct.

7-CHML of $A\left(T S_{4}\right)$ is shown in Figure 2.4.

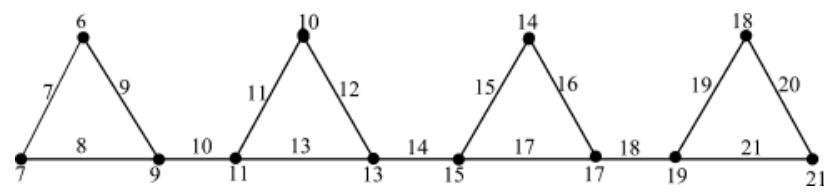

Figure 2.4. 4-CHML of $A\left(T S_{4}\right)$

\section{Theorem 2.3}

The graph $D A\left(T S_{n}\right)(n \geq 4)$ is a $k$-Contra Harmonic mean graph for any $k$.

\section{Proof}

Let $\left\{u_{l}, v_{l}, 1 \leq l \leq \frac{n}{2}, w_{l}, 1 \leq l \leq n\right\}$ be the vertices and $\left\{a_{l}, 1 \leq l \leq n-1, b_{l}, c_{l}, d_{l}, e_{l}, 1 \leq l \leq \frac{n}{2}\right\} \quad$ be $\quad$ the edges which are denoted as in Figure 2.5.

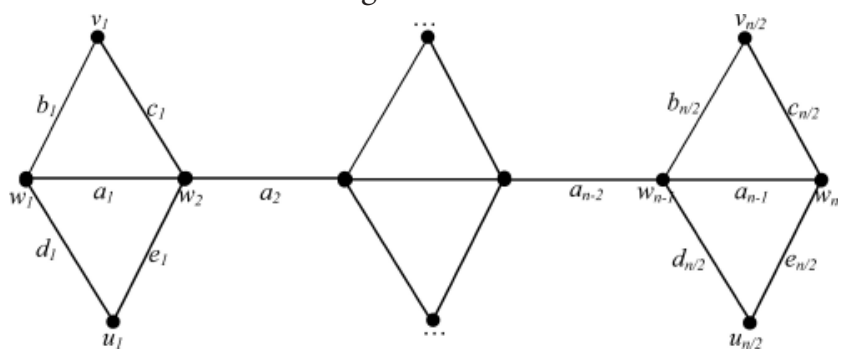

Figure 2.5: Ordinary labeling of $D A\left(T S_{n}\right)$

First we label the vertices:

For $1 \leq l \leq \frac{n}{2} f\left(v_{l}\right)=k+6 l-7 \quad f\left(u_{l}\right)=k+6 l-2$

$$
f\left(w_{l}\right)= \begin{cases}k+3 l-3 & \text { if } l \text { is odd } \\ k+3 l-4 & \text { if } l \text { is even }\end{cases}
$$

Then the induced edge labels are:

$$
f^{*}\left(a_{1}\right)=k+1
$$

For $2 \leq l \leq n-1 f^{*}\left(a_{l}\right)=k+3 l-1$

For $1 \leq l \leq \frac{n}{2} \quad f^{*}\left(b_{l}\right)=k+6 l-6$

$$
f^{*}\left(c_{1}\right)=k+2
$$

For $2 \leq l \leq \frac{n}{2} \quad f^{*}\left(c_{l}\right)=k+6 l-5$

$$
f^{*}\left(d_{1}\right)=k+4
$$

For $2 \leq l \leq \frac{n}{2} \quad f^{*}\left(d_{l}\right)=k+6 l-3$

$$
f^{*}\left(e_{1}\right)=k+3
$$

For $2 \leq l \leq \frac{n}{2} f^{*}\left(e_{l}\right)=k+6 l-2$

Hence, the edge labels are all distinct.

6-CHML of $D A\left(T S_{4}\right)$ shown in Figure 2.6. 


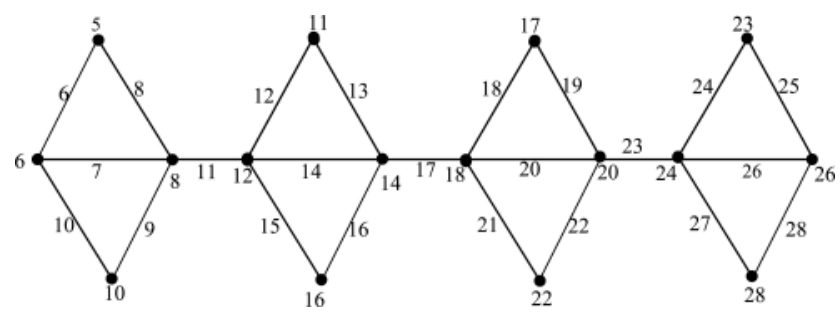

Figure 2.6. 4-CHML of $D A\left(T S_{4}\right)$

\section{Theorem 2.4}

The graph $Q S_{m}$ is a $k$-Contra Harmonic mean graph for any $k$.

\section{Proof}

Let $\left\{u_{l}, 1 \leq l \leq m, v_{l}, 1 \leq l \leq 2 m-2\right\} \quad$ be the vertices and $\left\{a_{l}, b_{l}, c_{l}, d_{l}, 1 \leq l \leq m-1\right\}$ be the edges which are denoted as in Figure 2.7.

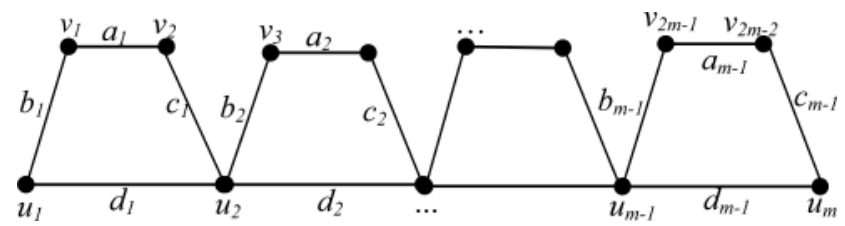

Figure 2.7. Ordinary labeling of $Q S_{m}$

First we label the vertices:

For $1 \leq l \leq 2 m-2 f\left(v_{l}\right)=k+2 l-2$

For $1 \leq l \leq m \quad f\left(u_{l}\right)=k+4 l-5$

Then the induced edge labels are:

$$
f^{*}\left(a_{1}\right)=k+3
$$

For $2 \leq l \leq m-1 \quad f^{*}\left(a_{l}\right)=k+4 l-2$

For $1 \leq l \leq m-1 \quad f^{*}\left(b_{l}\right)=k+4 l-4$

$$
f^{*}\left(c_{1}\right)=k+2
$$

For $2 \leq l \leq m-1 \quad f^{*}\left(c_{l}\right)=k+4 l-1$

For $1 \leq l \leq m-1 \quad f^{*}\left(d_{l}\right)=k+4 l-3$

Hence, the edge labels are all distinct.

2-CHML of $Q_{4}$ shown in Figure 2.8.

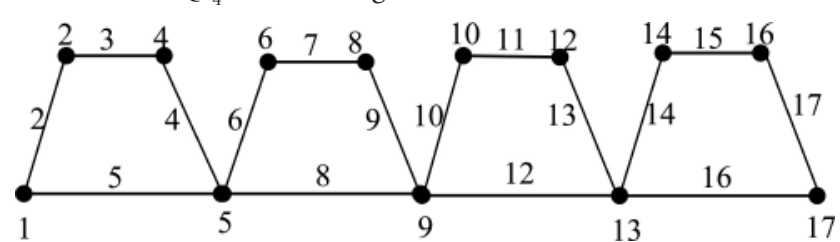

Figure 2.8. 4-CHML of $Q S_{4}$

\section{Theorem 2.5}

The graph $A\left(Q S_{m}\right)(m \geq 4)$ is a $k$-Contra Harmonic mean graph for any $k$.

\section{Proof}

Let $\quad\left\{u_{l}, v_{l}, 1 \leq l \leq m\right\} \quad$ be the vertices and $\left\{a_{l}, b_{l}, c_{l}, 1 \leq l \leq \frac{m}{2}, d_{l}, 1 \leq l \leq m-1\right\}$ be the edges which are denoted as in Figure 2.9.

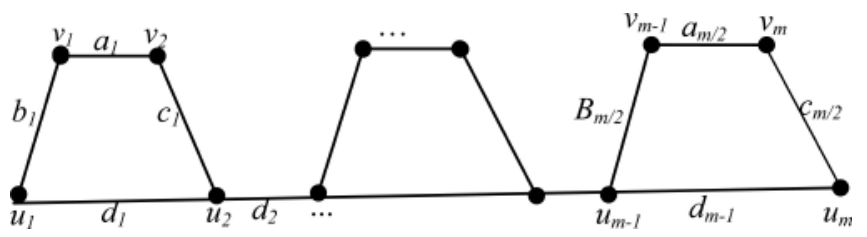

Figure 2.9. Ordinary labeling of $A\left(Q S_{m}\right)$

First we label the vertices:

For $1 \leq l \leq m \quad f\left(v_{l}\right)=k+4 l-5$

For $1 \leq l \leq m \quad f\left(u_{l}\right)=k+2(l-1)$

Then the induced edge labels are:

For $1 \leq l \leq \frac{m}{2} \quad f^{*}\left(a_{l}\right)=k+5 l-4 \quad f^{*}\left(b_{l}\right)=k+5(l-1)$

$$
f^{*}\left(c_{1}\right)=k+2
$$

For $2 \leq l \leq \frac{m}{2} \quad f^{*}\left(c_{i}\right)=k+5 i-2$

$$
f^{*}\left(d_{1}\right)=k+3
$$

For $2 \leq l \leq m-1$

$$
f^{*}\left(d_{l}\right)= \begin{cases}\frac{2 k+5 l-1}{2} & \text { if } l \text { is odd } \\ \frac{2 k+5 l-2}{2} & \text { if } l \text { is even }\end{cases}
$$

Hence, the edge labels are all distinct.

8-CHML of $A\left(Q S_{3}\right)$ is shown in Figure 2.10.

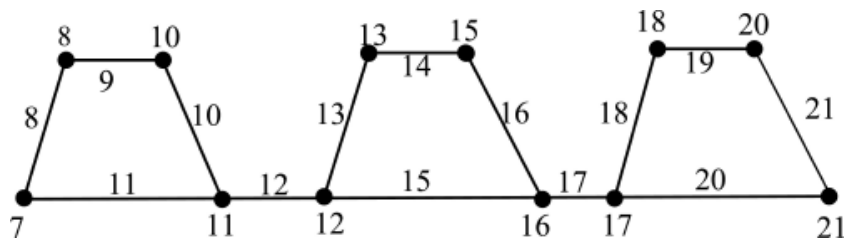

Figure 2.10. 4-CHML of $A\left(Q S_{3}\right)$ 


\section{References}

Gallian, J. A. (2018). A dynamic survey of Graph labeling, The Electronics Journal of Combinatorics, \#DS6.

Harary, F. (1988). Graph Theory, Narosa Publishing House Reading, New Delhi.
Narasimhan, G. Sampathkumar, R. (2013). Contra Harmonic Mean Labeling of Graphs, Mapana-Journal of Sciences, 12(8), 23-29.

https://doi.org/10.12723/mjs.26.7 